\title{
Manejo clínico de dientes supernumerarios en pacientes pediátricos
}

\author{
Jiménez de Sanabria \\ $G J$, Medina- $A C^{2}$ \\ Crespo-O $\mathrm{O}^{3}$ \\ Tovar- $R^{4}$
}

\section{Resumen}

Los dientes supernumerarios (DS) son aquellos que se forman adicionalmente a la serie normal. Pueden ubicarse en cualquier área de los maxilares, siendo su forma cónica, tuberculada o suplementaria. Las alteraciones de oclusión frecuentemente asociadas son desplazamiento, retardo de erupción o impactación de los dientes vecinos. Objetivo: Describir la prevalencia de dientes supernumerarios, así como las consecuencias y terapéutica implementada para un grupo de pacientes pediátricos. Material y métodos: fueron evaluados los registros diagnósticos de 823 pacientes del Servicio de Ortodoncia Interceptiva, postgrado de Odontología Infantil U.C.V., determinando: forma, ubicación, consecuencias y tratamiento efectuado; los pacientes con síndromes o HLP fueron excluidos. Resultados: La prevalencia de dientes supernumerarios fue 5,47\% con mayor proporción en el género masculino (1,8:1). La edad de diagnóstico fue 8,18 $( \pm 1,61)$. La forma cónica fue la más frecuente $(45 \%)$, siendo el área apical anterior la más afectada $(90 \%)$. El supernumerario produjo alteraciones en la posición de dientes adyacentes en 53,3\%. El tratamiento incluyó odontectomía $(63,3 \%)$ y conformación del arco dental $(87,5 \%)$. Sólo 4 casos requirieron tracción ortodóncica. Conclusión: la prevalencia de dientes supernumerarios en esta muestra fue mayor a la reportada por otros autores, con predilección por el género masculino. El tratamiento se realizó de forma sincronizada por parte del equipo interdisciplinario que incluyó Odontopediatra y Cirujano Bucal, determinando que la remoción oportuna del DS permite la erupción de los dientes adyacentes, siempre que el espacio disponible sea favorable.

Palabras clave: Dientes supernumerarios, prevalencia, tratamiento, pacientes pediátricos.

Artigo Original

\section{Manejo Clínico de dentes supranumerários em pacientes infantís}

\section{Resumo}

Os dentes supranumerários (DS), são aqueles que se formam adicionalmente à série dentária normal. Podem localizar-se em qualquer área dos maxilares, sendo a sua forma cônica, tuberculada ou suplementária. As alterações de oclusão frequentemnte associadas,são: desloca-

\footnotetext{
${ }^{1}$ Especialista en Odontología Infantil. Universidad Central de Venezuela

${ }^{2}$ Especialista en Odontología Infantil. Profesor Asociado, Departamento de Odontología Pediátrica y Ortodoncia, Universidad Central de Venezuela. caromemo@gmail.com

${ }^{3}$ Especialista en Ortodoncia. Profesor Agregado del Postgrado de Odontología Infantil, Universidad Central de Venezuela

${ }^{4}$ Especialista en Cirugía Bucal. Profesor asistente, Cátedra de Cirugía Bucal y Postgrado de Cirugía Bucal. Universidad Central de Venezuela.
} 
mento da posição original, retardo da erupção ou impactação dos dentes vizinhos. Objetivo: descrever a prevalência de dentes supranumerários, assim como as conseqüencias e terapêutica implementada para um grupo de pacientes infantís. Material e Metodo: foram avaliados os registros diagnósticos de 823 pacientes do Serviço de Ortodontia Interceptiva de Pósgraduação m Odontologia Infantíl U.C.V., determinando: forma, localização conseqüencias e tratamentos efetuados; os pacientes com síndromes ou HLP, foram excluídos. Resultados: A prevalência de dentes supranumerários foi de 5,47\% com maior proporção no gênero masculino, (1,8:1). A idade de diagnóstico foi de 8,18 (:+- 1,61). A forma cônica foi a mais freqüente $(45 \%)$, sendo a área apical anterior a mais afetada $(90 \%)$. O supra- numerário produziu alterações na posição dos dentes adjacentes em 53\%. O tratamento incluiu odontotomia $(63,3 \%)$ e coformação do arco dental $(87,5 \%)$. So 4 casos requeriram tração ortodontica. Conclusão: A prevalência de dentes supranumerários em nossa mostra foi maior que a reportada por outros autores, com prevlência maior para o gênero masculino. $O$ tratamento se realizou de forma sincronizada pela equipe interdisciplinar que incluiu Odontopediatra e Cirurgião Bucal, cdeterminano que a remoção oportuna do DS permite a erupção dos dentes adjacentes, sempre que o espaço disponível seja favorável.

Palavras Chaves: Dentes supranumerários; prevalência; tratamento; pacientes infantís.

\section{Original article}

\section{Clinical treatment for supernumerary teeth in pediatric patients}

\section{Abstract}

Supernumerary teeth (ST) are those formed additionally to the normal dental series. They may be found in any area of the maxilla or mandible with conical, tuberculate o supplementary shape. They may produce occlusal anomalies, and are associated with displacement, eruption disturbances or impaction of adjacent teeth. Objetive: The aim of this study is to describe frequency, associated anomalies and treatment outcome for ST in a group of pediatric patients, from Caracas, Venezuela. Material and methods: 823 orthodontic records (radiographs, photographs) of pediatric orthodontic patients were analyzed. ST shape and location were registered, as well as consequences on adjacent teeth and treatment outcome. Patients with syndromes, systemic disorders or clefts were excluded. Results: Frequency of ST was 5,47\%, with male: female ratio of 1.8:1. Mean age at diagnosis was $8.18( \pm 1.61)$. Conical shape was most frequent $(45 \%)$ and anterior apical area was the most affected (90\%). The $73,33 \%$ of all patients presented one ST, 22,22\% presented two and $4,44 \%$ three ST. Displacement of adjacent teeth was observed in 53.3\%. Treatment performed included extraction of ST (63.3\%) and space for adjacent teeth was gained by expansion and dental arch conformation (87.5\%). Spontaneous eruption of adjacent teeth occurred in all but 4 cases, which required orthodontic extrusion. Conclusion: Frequency of ST in this sample was 
higher than reported in other studies. Treatment outcome was favorable in most cases when extraction of ST and dental arch conformation were performed in the mixed dentition, by multidisciplinary team that included pediatric dentistry and oral surgeon.

Key words: Supernumerary teeth, prevalence, treatment, pediatric patients.

\section{Introducción}

El proceso de desarrollo dentario puede presentar diferentes alteraciones, ya sea en el número, forma o tamaño de los dientes. ${ }^{1}$ Entre las alteraciones de número, se encuentran los dientes adicionales al número normal y agenesias dentarias. $^{2,3}$ El término supernumerario se refiere a dientes adicionales ${ }^{3-6}$ también llamado hiperodoncia;, 3, 7-9 éstos pueden causar diferentes alteraciones en la conformación de los maxilares, superior e inferior, como apiñamiento, impactación de dientes permanentes, quistes dentígeros, desplazamiento de gérmenes dentarios y/o raíces y malposiciones dentarias; siendo una causa de maloclusiones. ${ }^{1,3}$ Suelen diagnosticarse en exámenes de rutina, clínicos y/o radiográficos, o en los casos cuyo motivo de consulta es la falla en la erupción de algún diente permanente. ${ }^{6,10}$

Los dientes supernumerarios se clasifican según su forma en cónico, suplementario y tuberculado. ${ }^{2,4,11}$ y según su ubicación en mesiodens, paramolar y distomolar. $2,7,11,12$

Su etiología es desconocida, 4,13,14 aunque se sabe que ocurre una duplicación de la lámina dental que da lugar a la aparición de órganos dentales adicionales. ${ }^{11,15}$ Han sido descritas diferentes teorías, de las cuales la más aceptada ha sido la Teoría de Hiperactividad de la Lámina Dental ${ }^{10,13-15}$ en la cual ocurre una proliferación del epitelio residual. ${ }^{10}$ Pueden presentarse como un fenómeno aislado o relacionado a síndromes como hendidura labio-palatina, Síndrome de Down, Displasia Cleidocraneal ${ }^{6}$, Síndrome de Gardner, entre otros. ${ }^{10,11,16}$

La prevalencia de los dientes supernumerarios es variable, entre $0,1 \%$ a $5,3 \%$, dependiendo de la población estudiada. La distribución de los mismos, así como las consecuencias que producen sobre la dentición en desarrollo y el tipo de tratamiento implementado, ha sido reportados en estudios internacionales. ${ }^{1,4,5,7,8,13,14,17}$

Los dientes supernumerarios suelen diagnosticarse como hallazgo en revisiones de rutina y en algunos casos el motivo de consulta es la falla de erupción de un diente permanente, $, 4,7,10,11,13$ en cualquiera de los casos las herramientas de diagnóstico son las radiografías panorámicas y oclusales, periapicales ${ }^{4,11,13,18}$ y más recientemente se ha descrito el uso de la tomografía computarizada Cone-Beam (imágenes 3D) para determinar la ubicación de los dientes supernumerarios y su relación con estructuras anatómicas adyacentes. ${ }^{19,20}$ Existen diferentes opciones de tratamiento que van desde la observación, control y guía de erupción, exodoncia, enucleación, ortodoncia; ;,8,11,13,17 el tratamiento deberá realizarse de forma individualizada en cada caso en particular, según las consecuencias de la presencia del diente supernumerario, etapa de la dentición, y características del o de los dientes supernumerarios presentes; para lo cual se requiere de la intervención de un equipo interdisciplinario conformado por odontopediatra, cirujano y ortodoncista. ${ }^{6}$

Pocos son los estudios que han evaluado detalladamente las consecuencias de la presencia de dientes supernumerarios y la evolución posttratamiento a largo plazo. Por lo que es necesa- 
rio evaluar cada caso y determinar el tratamiento más adecuado conforme a la evolución terapéutica observada. Además, se ha observado diferencias entre los resultados de los estudios alrededor del mundo, en parte por ser de poblaciones diferentes, con diferente alimentación, crecimiento, etnia, etc., así como por variaciones en las variables a evaluar en cada caso, y las posibles diferencias entre observadores. Todo esto, promueve la realización de estudios en la población pediátrica de Venezuela a fin de determinar la prevalencia, distribución demográfica, consecuencias y terapéutica a implementar, propia de su población.

\section{Material y métodos}

Este estudio se recibió el aval del Comité de Bioética de la Facultad de Odontología de la Universidad Central de Venezuela Nº 0197-2011.

Es una investigación teórico-documental y de campo, descriptiva, correlacional, longitudinal y retrospectivo. La población estuvo conformada por las historias y registros de los pacientes atendidos en el servicio de Ortodoncia Interceptiva del Postgrado de Odontología Infantil de la UCV entre 2001-2010, siendo en total de 823 pacientes. La muestra se obtuvo a través de un muestreo no probalístico, ya que los pacientes fueron escogidos a conveniencia. Los criterios de inclusión fueron: presencia de dientes supernumerarios de dientes permanentes, registros radiográficos completos, historia clínica completa con datos médicos y la secuencia de tratamiento. Fueron excluidos los casos con radiografías en mal estado que impidieran el correcto diagnóstico, fallas en la anotación de secuencia de tratamiento y pacientes con síndromes o alteraciones genéticas.

Se excluyeron 4 pacientes por presentar síndromes (2 con Displasia Cleidocraneal, 1 Síndrome de Crouzon, 1 Síndrome de Gorlin-Goltz) y 2 pacientes por presentar Hendidura de Labio y Paladar, 7 pacientes con registros incompletos quedando conformada por 45 pacientes. De los dientes supernumerarios encontrados en los 45 pacientes de la muestra, 3 se excluyeron por ser de dientes primarios, por lo que de 63 dientes supernumerarios, la muestra quedó conformada por 60 dientes supernumerarios de dientes permanentes.

Las radiografías panorámicas se evaluaron utilizando negatoscopio, identificando el o los dientes supernumerarios. Se registraron las características como forma, ubicación (bases apicales descritas por van den Linden (21)), erupción (erupcionado, no erupcionado e invertido), consecuencias en los dientes adyacentes (desplazamiento, rotación, desplazamiento de más de 2 dientes); también se registraron los datos de secuencia de tratamiento para determinar el tipo de tratamiento quirúrgico y ortodóncico, junto con la fecha de realización de cada uno.

La edad fue determinada restando la fecha de nacimiento de la fecha de la radiografía o la realización del tratamiento.

Se procedió a realizar la transcripción y tabulación de los datos a través del programa Microsoft Office Excel 2007. Los procedimientos estadísticos fueron realizados mediante el programa SPSS para Windows versión 18 en el módulo de análisis estadístico de frecuencia, descriptivos, tablas de contingencia y T Student $(p=0,05)$.

\section{Resultados}

La prevalencia de pacientes con DS fue de 5,47\% $(\mathrm{N}=45)$ de la población evaluada. La edad media fue 8,18 años (+/ - 1,61). La distribución en cuanto al género de los pacientes que presentaron 


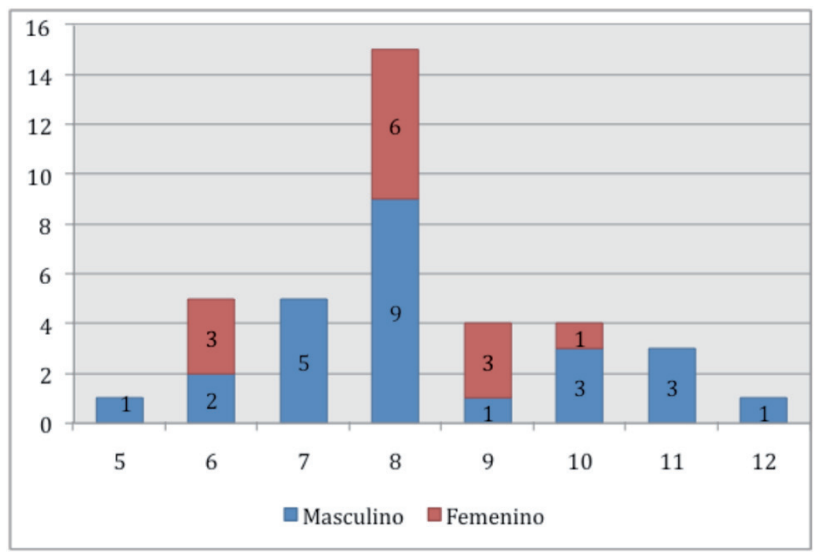

Figura 1. Gráfico de frecuencia por grupo de edad.

dientes supernumerarios, arrojó un porcentaje de $64,4 \%$ para masculino y $35,6 \%$ femenino. Con una proporción de 1,8:1 (Masculino:Femenino). (Fig. 1).
No se halló diferencia estadísticamente significativa en la media de dientes supernumerarios por paciente entre géneros al género (prueba $\mathrm{T}$ de Student, $\mathrm{p}=0,05$ ) entre los grupos.

La frecuencia según el maxilar y la zona del maxilar (área apical) donde se encuentra el supernumerario se muestra en la Tabla 1.

La forma más frecuentemente hallada fue cónica $(45 \%$, ) seguida de tuberculado $(35 \%)$ y suplementario $(20 \%)$. En cuanto a la relación entre la forma y la erupción de los dientes supernumerarios, la mayoría de los dientes supernumerarios no erupcionados son tuberculados, siendo 16, y solo 4 tuberculados erupcionaron (Tabla 2).

Tabla 1. Distribución según el área apical y maxilar.

\begin{tabular}{|c|c|c|c|c|c|c|}
\hline & Maxilar superior & \multicolumn{2}{|c|}{ Mandíbula } & \multicolumn{2}{|c|}{ Total } \\
\hline Área apical & $\mathrm{N}$ & $\%$ & $\mathrm{~N}$ & $\%$ & $\mathrm{~N}$ & $\%$ \\
\hline Anterior & 52 & 86,67 & 2 & 3,33 & 54 & 90,0 \\
\hline Media & 1 & 1,67 & 2 & 3,33 & 3 & 5,0 \\
\hline Posterior & 3 & 5,00 & 0 & 0 & 3 & 5,0 \\
\hline Total & 56 & 93,34 & 4 & 6,66 & 60 & 100,0 \\
\hline
\end{tabular}

Tabla 2. Relación entre forma y erupción de los dientes supernumerarios.

\begin{tabular}{|c|c|c|c|c|c|c|c|c|}
\hline \multirow[b]{3}{*}{ Forma } & \multicolumn{6}{|c|}{ Erupción de los dientes supernumerarios } & \multirow{2}{*}{\multicolumn{2}{|c|}{ Forma }} \\
\hline & \multicolumn{2}{|c|}{ No } & \multicolumn{2}{|c|}{ Invertido } & \multicolumn{2}{|c|}{ Sí } & & \\
\hline & $\mathrm{N}$ & $\%$ & $\mathrm{~N}$ & $\%$ & $\mathrm{~N}$ & $\%$ & $\mathrm{~N}$ & $\%$ \\
\hline Cónico & 5 & 8,48 & 13 & 22,03 & 9 & 15,25 & 27 & 45,00 \\
\hline Suplementario & 6 & 10,17 & 0 & 0 & 6 & 10,17 & 12 & 20,00 \\
\hline Tuberculado & 16 & 27,12 & 0 & 0 & 4 & 6,78 & 21 & 35,00 \\
\hline Total & 28 & 45,77 & 13 & 22,03 & 19 & 32,2 & 60 & 100 \\
\hline Total no erupcionados & \multicolumn{4}{|c|}{$67,79 \%$} & & & & \\
\hline
\end{tabular}


En cuanto a la relación entre la localización y forma del supernumerario, en el maxilar superior se hallaron 27 supernumerarios cónicos y 19 tuberculados. Al evaluar la relación entre la forma y la ubicación de los dientes supernumerarios presentes, el más común fue el diente supernumerario cónico ubicado en el área apical anterior, 45\% ( $\mathrm{N}=27)$. El 100\% de los cónicos se encontró en el área apical anterior. Los supernumerarios suplementarios de hallaron en el área apical anterior y media. Los supernumerarios tuberculados fueron hallados en todas las zonas de los maxilares. (Fig. 2).

Al distribuir los dientes supernumerarios conforme a la cantidad por cada paciente, se determinó que $33(73,33 \%)$ pacientes presentaron 1 supernumerario, $10(22,22 \%)$ presentaron 2 dientes supernumerarios y sólo 3 presentaron 3 $(4,44 \%)$ dientes supernumerarios.

De los 60 dientes supernumerarios encontrados, se evaluaron la consecuencias de éstos en el área apical correspondiente. En cuanto al tipo y fecha de tratamiento quirúrgico, 11 casos debieron ser excluidos por abandono de tratamiento o fallas en la anotación de secuencia de tratamiento; por lo que en esta variable la muestra fue de 49 dientes supernumerarios.

Las consecuencias sobre la posición de los dientes adyacentes se detallan en las Fig. 3 y Fig. 4. El 45\% presentó retardo de erupción asociado a la presencia de DS. El 53\% de los dientes adyacentes al DS presentó algún tipo de alteración de posición, siendo más frecuente el desplazamiento $(25 \%)$ y las rotaciones $(20 \%)$.

En cuanto al tipo de cirugía realizada, el 63,3\% de los dientes supernumerarios se trató con odontectomía, 17 dientes $(34,7 \%)$ fueron extraídos por medio de exodoncia simple y 1 diente suplementario permaneció en boca por estar correctamente alineado. La edad media en que se realizó la cirugía fue de 9,47 años $( \pm 1,45)$.

En cuanto a la frecuencia del tipo de tratamiento ortodóncico, la mayor proporción estuvo enfocado a conformar el arco por medio de expan-

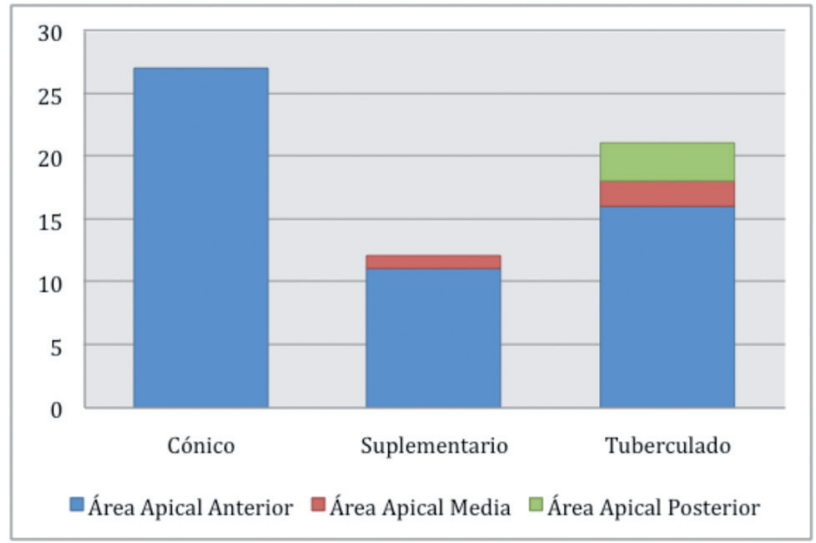

Figura 2. Relación entre forma y área apical de los dientes supernumerarios.

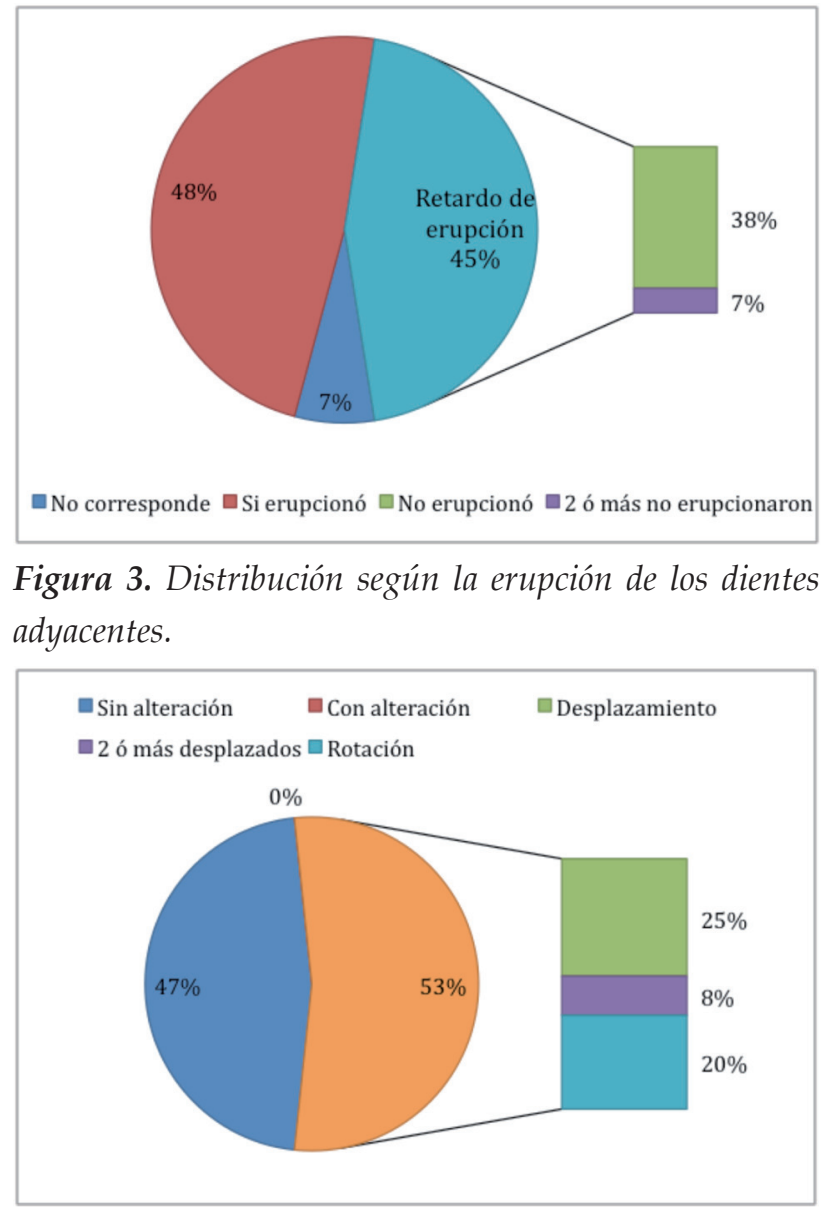

Figura 4. Consecuencias sobre los dientes adyacentes. 
sión y mantenimiento de espacio, en un $87,5 \%$ $(\mathrm{N}=42)$ de los casos, seguido de 8,3\% (N=4) tracción ortodóncica y en el 4,2\% (N=2) no se realizó ningún tratamiento ortodóncico.

La edad media de la instalación de aparatos fue a los 9,46 años, $( \pm 1,89)$. Además se distribuyó la instalación de los aparatos conforme a la edad, siendo más frecuente la instalación de aparatos a los 8 años en un 24,3\% de los casos. (Fig. 5).

Se relacionó la erupción de los dientes adyacentes al supernumerario con el tratamiento ortodóncico implementado, resultando que el tratamiento más común fue la conformación de arco, aún en los casos donde había falla o retardo de erupción de los dientes adyacentes. Y solo en 4 casos de los 17 que presentaron falla de erup-

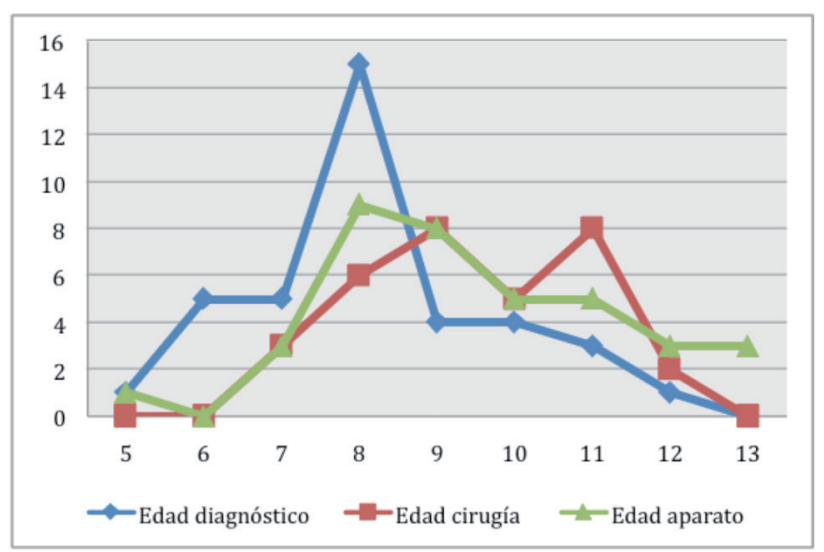

Figura 5. Distribución de la edad de diagnóstico, cirugía e instalación de aparatos según la edad. ción, fue necesario realizar tracción ortodóncica. En los 4 casos donde 2 ó más dientes no erupcionaron se realizó tracción ortodóncica. En la Tabla 3 se detalla la relación entre la forma de los dientes supernumerarios y el tratamiento ortodóncico implementado.

\section{Discusión}

Los dientes supernumerarios se presentaron en un $5,47 \%$ ( $n=45$ pacientes) de la población estudiada, ligeramente superior a lo reportado por otros estudios en Venezuela ${ }^{21,22}$ e internacionales $^{1,3-5,8,10,11,15}$. Esto puede deberse a que la población estudiada pertenece al servicio de Ortodoncia Interceptiva, siendo la falla de erupción causada por la presencia de SD el motivo de consulta.

Se determinó la frecuencia de la cantidad de supernumerarios por pacientes, la cual resultó ser más común la presencia de 1 supernumerario único en un $75 \%$, seguido de 2 supernumerarios, y en menor proporción 3 y más supernumerarios, este orden es similar al reportado en estudios realizados en niños sin síndromes $^{1,4,10,11,13,21,-26}$.

La edad media de los pacientes con dientes supernumerarios al momento del diagnóstico fue

Tabla 3. Frecuencia según forma del supernumerario y tratamiento ortodóncico.

\begin{tabular}{|c|c|c|c|c|}
\hline \multirow{2}{*}{ Forma } & \multicolumn{3}{|c|}{ Tipo de tratamiento ortodóncico } & \multirow{2}{*}{ Total } \\
\cline { 2 - 5 } & Ninguna & Conformación de Arco & Tracción ortodóncica & \\
\hline Cónico & 1 & 22 & 1 & 24 \\
\hline Suplementario & 1 & 6 & 1 & 8 \\
\hline Tuberculado & 0 & 14 & 4 & 48 \\
\hline Total & 2 & 42 & & 4 \\
\hline
\end{tabular}


de 8,18, correspondiendo a la primera etapa de dentición mixta, cuando pueden producirse alteraciones en la erupción o los pacientes son evaluados por maloclusiones que ya pueden ser clínicamente apreciadas, complementando el diagnóstico con la indicación de realización de radiografías.

La distribución por género de los pacientes con supernumerarios se presentó con mayor frecuencia en el masculino, en un $64,4 \%$ de los pacientes, con una proporción de 1,8:1 (Masculino:Femenino) lo cual concuerda con lo reportado previamente en la literatura. ${ }^{1,4,5,7,8,10,11,13,22}$ No hubo diferencia estadísticamente significativa entre la media de cantidad de dientes supernumerarios y el género.

Los supernumerarios se clasificaron según su forma, siendo el cónico $(45,0 \%)$ el más frecuente en este estudio, seguido del tuberculado $(35,0 \%)$ y en menor porcentaje el suplementario (20\%); cuyo orden es similar a algunos estudios., ${ }^{5,13,21}$ Mientras que Esenlik y Cols. ${ }^{4}$ Anthonappa y Cols. ${ }^{7}$ y Schmuckli y Cols. ${ }^{10}$ reportaron igualmente el cónico como más frecuente, pero seguido del suplementario y por último el tuberculado.

El 93,3\% de los dientes supernumerarios se localizó en maxiilar superior, en concordancia con otros estudios. ${ }^{1,15}$ Según el área apical, la mayor frecuencia fue en la anterior presentándose en un $90,0 \%$, en concordancia con estudios en otras poblaciones. ${ }^{1,4,5,7,10}$

El mayor porcentaje de supernumerarios no erupcionó, esto incluye a los que no erupcionaron y a los que se encontraban invertidos, representando un $67,79 \%$, resultado similar a lo descrito en pacientes asiáticos, ${ }^{7}$ europeos, ${ }^{4,10,15}$ y latinoamericanos. ${ }^{1,21}$ De todos los dientes su- pernumerarios evaluados la mayoría de los no erupcionados son tuberculados $27,12 \%$. Sin embargo, a diferencia de lo planteado por otros autores, ${ }^{13}$ sí hubo DS tuberculados que lograron erupcionar $(6,78 \%)$.

Al relacionar la forma del supernumerario con la localización, resultó ser el cónico el más común en el maxilar superior, seguido del tuberculado; y en la mandíbula el suplementario. No se encontraron reportes de esta relación. Sin embargo, en cuanto a la relación entre la forma y el área apical, Schmuckli y Cols. ${ }^{10}$ y Calvano y Cols. ${ }^{5}$ obtuvieron que el diente cónico fue el más común en el área apical anterior, similar a los resultados obtenidos en este estudio, donde el $100 \%$ de los DS cónicos se encontraron en el área apical anterior.

Relacionando la localización con el área apical la zona más común de presentación de supernumerarios fue el área apical antero-superior $(86,67 \%)$, en concordancia con otros estudios, ${ }^{1,4,7,10,21}$ y diferente a lo reportado por Leco y Cols. ${ }^{15}$ quienes destacan el área apical posterior como la más común en el maxilar superior. Esto puede relacionarse con la edad de la población. En adultos puede haber formación tardía de dientes supernumerarios en la zona molar (paramolares y distomolares). Esta muestra estuvo compuesta exclusivamente por pacientes pediátricos.

La presencia de uno o más dientes supernumerarios acarrea diversas consecuencias en el desarrollo de la oclusión, en la muestra evaluada la consecuencia más frecuente fue las alteraciones de posición de los dientes adyacentes, como rotación, desplazamiento, lo que concuerda con lo reportado por Leco y Cols. ${ }^{15}$ seguido del retardo de erupción de los dientes adyacentes. En la mayoría de los casos no hubo alteración 
de los dientes adyacentes en posición ni erupción lo cual concuerda con lo reportado por Anthonappa y Cols. ${ }^{7}$

En cuanto al tratamiento quirúrgico realizado, la cirugía más común fue la odontectomía, 63,3\%, ésto debido a que la mayoría de los dientes estaban incluidos; siendo la edad media al momento de la cirugía fue 9,47 años $( \pm 1,45)$, igual a lo reportado por Patchett y cols. ${ }^{13}$ CahuanaCárdenas y Cols., ${ }^{17}$ y mayor a lo reportado por Anthonappa y Cols. ${ }^{7}$, lo cual concuerda con la edad cercana, pero anterior, al cierre apical de los incisivos.

En cuanto a la erupción de dientes adyacentes y el tipo de tratamiento ortodóncico, de los 4 casos que requirieron tracción ortodóncica, 2 tuvieron 1 supernumerario tuberculado, que no erupcionó, que impedía la erupción de por lo menos 1 diente permanente y que el espacio disponible en el arco era suficiente; lo que con- cuerda con lo reportado por Patchett y cols., ${ }^{13}$ quien reportó que más del $60 \%$ de los dientes permanentes asociados a un supernumerario tuberculado requirió tratamiento quirúrgicoortodóncico. Cabe destacar que la edad a la cual se realizó la cirugía de estos supernumerarios tuberculados, fue entre los 10 años +10 meses y 11 años +4 meses, lo cual concuerda con lo reportado por Cahuana-Cárdenas y cols. ${ }^{17}$ que obtuvo en la mayoría de los casos, donde la cirugía del diente supernumerario se realizó a partir de los 10 años, requirieron exposición quirúrgica y/o tracción ortodóncica. Esto demuestra la importancia de que se realice el análisis integral de la maloclusión, y luego la cirugía en el momento adecuado, considerando, además de las características del supernumerario, la etapa de desarrollo de la dentición del paciente. Esto se logra con el trabajo del equipo interdisciplinario conformado por Odontopediatra, Ortodoncista y Cirujano Bucal.

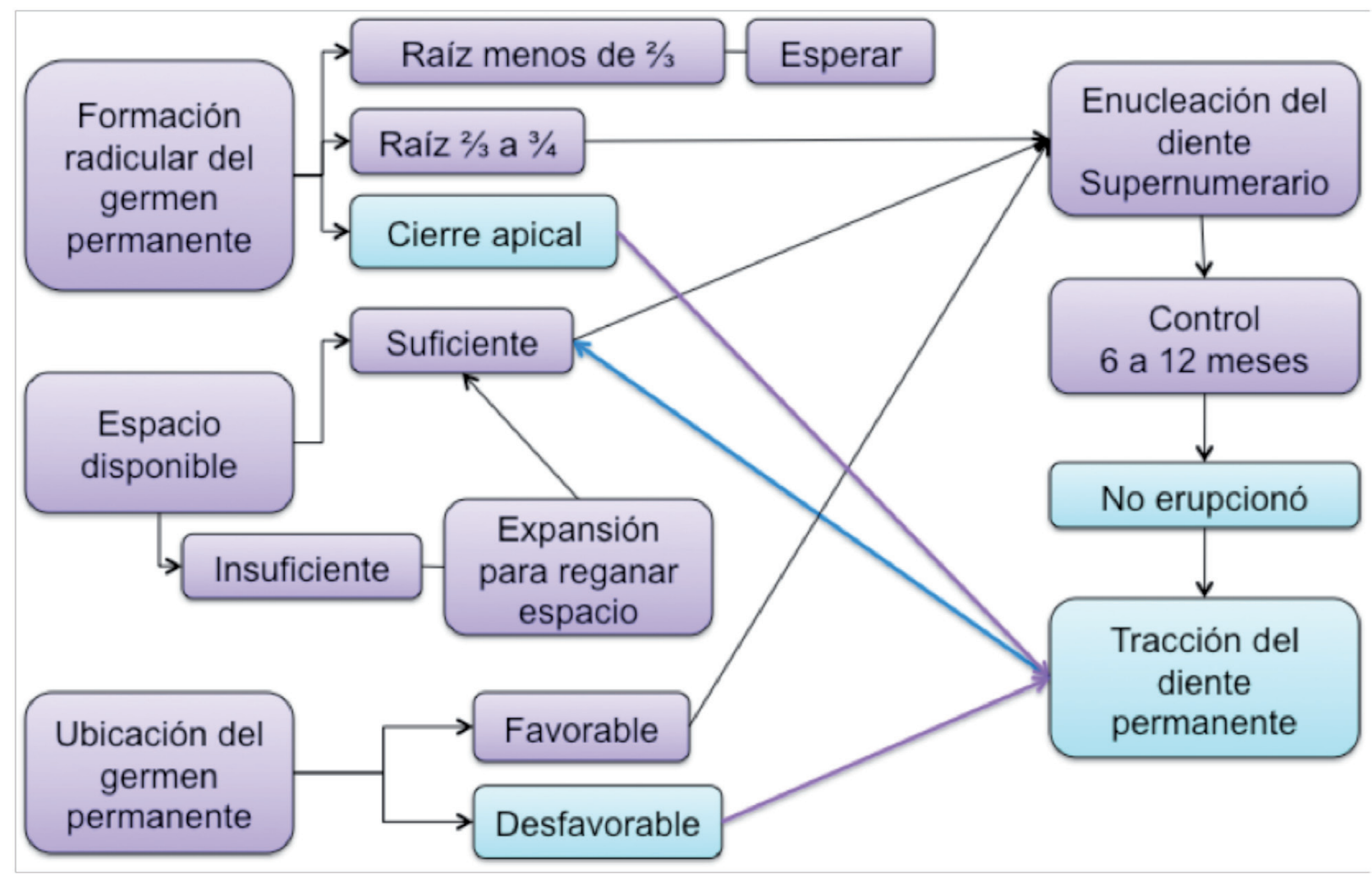

Figura 6. Representación esquemática del protocolo de atención para pacientes pediátricos con dientes supernumerarios. 
El tipo de tratamiento más frecuente fue aquel enfocado a la conformación de arco, como expansores y mantenedores de espacio, seguido de tracción en menor proporción; y los aparatos más utilizados fueron las placas removibles activas, seguido de aparatos fijos de diferentes diseños; con una edad media de colocación similar a la de la cirugía. Estos aspectos se deben a que la población estudiada pertenece al servicio de Ortodoncia Interceptiva, donde los objetivos de tratamiento primordiales son fomentar el desarrollo normal, funcional y estético, de la oclusión. Igualmente, la planificación terapéutica se realiza de forma interdisciplinaria, incluyendo al cirujano bucal. En estos casos se procura la planificación quirúrgica en sincronía con la ortodóncica, optimizando los resultados terapéuticos. En este sentido, la planificación de le remoción del diente supernumerario se realiza de forma oportuna, realizando el abordaje quirúrgico cuando los dientes adyascentes presenten $\mathrm{de}^{2} / 3$ a $3 / 4$ de formación radicular. Esto permite estimar que aún tienen potencial de erupción y que, además, la cantidad de raíz formada es apropiada para su emergencia en boca. El protocolo de tratamiento implementado se detalla en la Fig. 6.

El tipo de tratamiento ortodóncico realizado en los pacientes pediátricos está enfocado a guiar la erupción, aminorar las consecuencias y optimi- zar los períodos de erupción y desarrollo de la dentición. Esto se ve reflejado en que, al eliminar los Desde la zona apical anterior, a la edad de 8 a 9 años aproximadamente, los dientes adyacentes lograron erupcionar sin necesidad de tracción.

\section{Conclusiones}

La prevalencia de dientes supernumerarios en esta población fue $5,47 \%$, mostrando una predilección por el género masculino 1,8:1, ubicándose más comúnmente en el maxilar superior, en el área apical anterior, como un DS único. La forma más frecuente fue cónica, seguida por el tuberculada. Los DS frecuentemente no erupcionan $(67,79 \%)$, por lo cual en algunos casos suelen causar retardo de erupción de los dientes adyacentes.

La odontectomía oportuna de los dientes supernumerarios no erupcionados, permite la erupción de los dientes adyacentes, siempre que las condiciones en el arco dental sean favorables.

Para evitar y tratar las alteraciones causadas por los dientes supernumerarios se requiere de la participación del odontopediatra, ortodoncista y cirujano bucal, formando así el equipo interdisciplinario que es necesario para el manejo adecuado de cada caso.

\section{Referencias}

1. Chappuzeau E, Cortés D. Anomalías de la Dentición en Desarollo: Agenesias y Supernumerarios. Revista Dental de Chile, 2008; 99 (2): 3-8.

2. Canut JA. Ortodoncia Clínica y Terapéutica. Segunda edición. España. Editorial Masson. 2000.

3. Méndez DC, Contreras CC. Anomalías de número en pacientes con dentición mixta. Rev Latinoam Orto Odontoped, Internet 2006: 1-5. Disponible en http: / / www.ortodoncia.ws/ publicaciones/2006/ art3.asp

4. Esenlik E, Özgür Sayin M, Onur Atilla A, Özen T, Altun C, Basak F. Supernumerary teeth in a Turkish population. Am J Orthod Dentofacial Orthop. 2009;136: 848-52.

5. Calvano E, Gomes A, De Castro Costa M. Rezende A, Granjeiro JM. Supernumerary teeth vary depending on gender. Braz Oral Res. 2011; 25(1):76-9. 
6. Mukhopadhyay S. Mesiodens: A clinical and radiographic study in children. J Ind Soc Pedo Prev Dent, 2011; 29(1): $34-8$. 7. Anthonappa RP, Omer, RSM, King NM. Characteristics of 283 supernumerary teeth in southern Chinese children. Hong Kong. Oral Surg Oral Med Oral Pathol Oral Radiol Endod. 2008; 105(6): e48-54.

8. Discacciati MS, Lértora MF. Anomalías Dentarias: Prevalencia Observada Clínicamente, en niños de la Ciudad de Corrientes. Argentina. UNIVERSIDAD NACIONAL DEL NORDESTE; Internet 2007, Disponible en http:/ / www.unne.edu. ar/Web / cyt/ com2005/3-Medicina/M-028.pdf acceso 6-4-12.

9. Mevlut C, Hasan K, Hüsamettin O. Prevalence and characteristics of supernumerary teeth in a non-syndrome Turkish population: Associated pathologies and proposed treatment. Med Oral Patol Cir Bucal. 2010; 15(4): e575-8.

10. Schmuckli R, Lipowski C, Peltomäki T. Prevalence and Morphology of Supernumerary Teeth in the population of a Swiss Community. Schweiz Monatsschr Zahnmed. 2010; 11: 987-90.

11. Salcido-García JF, Ledesma-Montes C, Hernández-Flores F, Pérez D, Garcés-Ortiz M. Frecuencia de dientes supernumerarios en una población mexicana. Med Oral Patol Oral Cir Bucal. 2004; 9 (5): 403-9.

12. Rodríguez Romero F, Cerviño Ferradanes S. Múltiples dientes supernumerarios distomolares. Av. Odontoestomatol. 2009; 26(6): p. 319-25.

13. Patchett CL, Crawford PJM, Cameron AC, Stephens CD. The management of supernumerary teeth in chilhood - a retrospective study of practice in Bristol Dental Hospital, England and Westmead Dental Hospital, Sydney, Australia. Int J Paediatr Dent. 2001; 11: 259-65.

14. Yaguë-García J, Berini-Aytés L, Gay-Escoda C. Múltiple supernumerary teeth not associated with complex syndromes: A retrospecive study. Med Oral Patol Oral Cir Bucal. 2009; 14(7): E331-6.

15. Leco MI, Martín M JF, Martínez JM. An observational study of the frecuancy of supernumerary teeth in a population of 2000 patients. Med Oral Patol Oral Cir Bucal. 2007; 12: E134-8.

16. Peker I, Kaya E, Darendeliler-Yaman S. Clinical and radiographical evaluation of non-syndromic hypodontia and hyperdontia in permanent dentition. Med Oral Patol Oral Cir Bucal. 2009;14(8): e393-7.

17. Cahuana-Cárdenas A, Alfaro A, Pérez B, Coelho A. Dientes supernumerarios anteriores no erupcionados: revisión de 125 casos. RCOE. 2003; 8(3): 263-71.

18. Garvey MT, Barry HJ, Blake M. Supernumerary Teeth-An Overview of Classification, Diagnosis and Management. J Can Dent Assoc. 1999; 65: 612-6.

19. Katheria BC, Kau CH, Tate R, Chen JW, English J, Bouquot J. Effectiveness of impacted and supernumerary tooth diagnosis from traditional radiography versus cone beam computed tomography. Pediatr Dent. 2010; 32(4): 304-9.

20. Liu DG, Zhang WL, Zhang ZY, Wu YT, Ma XC. Three-dimensional evaluations of supernumerary teeth using conebeam computed tomography for 487 cases. Oral Surg Oral Med Oral Pathol Oral Radiol Endod. 2007; 103(3):403-11.

21. Medina AC, Martínez MG. Supernumerary teeth in an urban Venezuelan population. Int J Paediatr Dent. 2011; 21(1): 203.

22. Iglesias P, Manzanares MC, Valdina I, Zambrano R, Solórzano E, Tallón V, Carvalho P. Anomalías dentarias: prevalencia en relación con patologías sistémicas en una población infantil de Mérida, Venezuela. Revista Odontológica de Los Andes, 2007; 2,(2): 37-50.

23. Fernández P, Valmaseda E, Berini L, Gay C. Estudio retrospectivo de 145 dientes supernumerarios. Med Oral Patol Oral Cir Bucal 2006; 11: e339-44.

24. Ferrés-Padró E, Prats-Armengol J, Ferrés-Amat E. A descriptive study of 113 uneupted supernumerary teeth in 79 pediatric patients in Barcelona. Med Oral Patol Oral Cir Bucal. 2009 Mar 1; 14(3): p. E146-52.

25. Günduz K, Çelenk P, Zengin Z, Sümer P. Mesiodens: a radiographic study in children. Journal of Oral Science. 2008; 50(3): p. 287-91.

26. Gallas M, García A. Retention of prmanent incisors by mesiodens: a family affair. Br Dent J. 2000 Enero 22; 188(2): p. $63-4$.

Texto traducido por: Antonio J. Nogueira

Recibido: 18-03-12

Aceptado: 22-04-12

Correspondencia: gabrielajbrito@gmail.com 